\title{
A Rapid Library Screen for Tailoring $\beta$-Peptide Structure and Function
}

\author{
Joshua A. Kritzer ${ }^{\dagger}$, Nathan W. Luedtke ${ }^{\dagger}$, Elizabeth A. Harker ${ }^{\dagger}$, and Alanna Schepartz ${ }^{\star}, \dagger, \ddagger$ \\ Departments of Chemistry and Molecular, Cellular, and Developmental Biology, Yale University, \\ New Haven, Connecticut 06520
}

Recently we described the $\beta$-decapeptide $\boldsymbol{\beta 5 3 - 1}$ (Figure 1), which folds into a 14-helix, binds the oncoprotein hDM2 with submicromolar affinity, and inhibits the interaction of hDM2 with a peptide derived from the activation domain of $\mathrm{p} 53$ (p53AD). ${ }^{1}$ The solution structure of ק53-1 in $\mathrm{CD}_{3} \mathrm{OH}$ revealed an unexpected $\mathrm{C}$-terminal unwinding that staggers the side chains comprising the $\mathrm{hDM} 2$ recognition epitope to better mimic those of p53AD., ${ }^{2,3}$ The structurefunction relationship implied by this distortion suggested that a library of $\boldsymbol{\beta 5 3 - 1}$ analogues with diversity along a nonrecognition face might contain more active analogues. Here we describe (1) $\beta$-peptide synthesis protocols that produce high-quality one-bead-one- $\beta$-peptide (OBO $\beta$ ) libraries suitable for on-bead screening without purification, ${ }^{4}(2)$ a scalable on-bead screen, and (3) a simple tandem mass spectrometry (MS/MS) decoding method. Using this procedure, we identified $\boldsymbol{\beta 5 3 - 1}$ analogues with improved structural and functional properties.

Previous reports have documented poor yields and purities in the synthesis of $\beta$-peptide oligomers longer than hexamers, making HPLC purification a prerequisite for analysis..$^{5-8}$ To evaluate conditions that would produce high-quality $\beta^{3}$-peptide decamers suitable for analysis without purification, we synthesized $\boldsymbol{\beta 5 3 - 1}$ and $\beta$ NEG (Figure 1) on Tentagel macrobeads containing a 4-hydroxy-methylbenzoic acid (HMBA) handle. Synthesis products were deprotected (96:2:2 TFA/water/triisopropylsilane), and pools of 100-200 beads were cleaved $\left(1.0 \mathrm{M} \mathrm{CH}_{3} \mathrm{ONa} / \mathrm{MeOH}\right)$ for bulk purity analysis. We found that a combination of 90-min coupling times, double couplings, and an extra Fmoc deprotection step using DBU after the fourth coupling 7,9 yielded bead-bound $\beta$-decapeptides with purities between 80 and $90 \%$ as judged by analytical HPLC. The purities of $\beta$-peptides isolated from individual $\beta$ 53-1 and $\boldsymbol{\beta}$ NEG beads were evaluated by MALDI and tandem electrospray (MS/MS) mass spectrometry; in each case a single major product was observed. Overall, this refined protocol produces $\beta$-peptide libraries whose quality matches or exceeds those of previously reported peptoid and oligocarbamate libraries ${ }^{10-15}$ and equals the purities reported for microwaveassisted $\beta$-peptide syntheses. ${ }^{8}$

To establish a procedure for on-bead screening, we used $\boldsymbol{\beta 5 3 - 1}$ and $\boldsymbol{\beta}$ NEG beads synthesized using the refined protocols as positive and negative controls for $\mathrm{hDM} 2_{1-188}$ binding. The screen entailed blocking, incubation with biotinylated hDM $2_{1-188}\left({ }^{(B i} \mathrm{hDM} 2\right)$, washing, incubation with streptavidin-coated quantum dots (QDots-SA605), further washing, and visualization using a fluorescence microscope. Detection using streptavidin-coated quantum dots eliminates false positives due to Tentagel bead autofluorescence. ${ }^{12,16}$ Exhaustive testing of buffers and

\footnotetext{
alanna.schepartz@yale.edu.

tDepartment of Chemistry.

末Department of Molecular, Cellular, and Developmental Biology.

Supporting Information Available: $\beta$-peptide synthesis and screening protocols, CD spectra, and NMR structure determination details. This material is available free of charge via the Internet at http://pubs.acs.org.
} 
additives indicated that a blocking and washing buffer (BW buffer) composed of $10 \mathrm{mM}$ Tris$\mathrm{HCl}$ (pH 8.0), $2.5 \mathrm{mg} / \mathrm{mL}$ gelatin, $0.15 \mathrm{M} \mathrm{NaCl}$, and $0.1 \%$ Tween- 20 prevented localization of quantum dot fluorescence to $\beta$ NEG beads while maintaining signal on $\boldsymbol{\beta 5 3 - 1}$ beads (Figure $2 \mathrm{~A})$. Notably, decreasing BiohDM2 concentration and increasing wash number and duration or the concentrations of gelatin, $\mathrm{NaCl}$, and/or Tween-20 in the blocking and washing buffer each attenuated signal intensity. ${ }^{9}$ The sensitivity of the screen to multiple parameters indicates that it can be tuned in several independent ways to achieve a desired hit rate. ${ }^{17}$

As a mock screen, we chose one high-intensity bead from a mixture of five $\boldsymbol{\beta 5 3 - 1}$ beads and a vast excess (>1000) of $\boldsymbol{\beta}$ NEG beads. The bead was washed and cleaved, and the cleaved products were desalted, ${ }^{9}$ theoretically yielding up to $0.7 \mathrm{nmol} \beta$-peptide. Robust MALDI spectra revealing a single major product were obtained using only $5 \%$ of this material. MS/MS spectra were obtained using less than half of the remaining material, ${ }^{9}$ highlighting the ease with which high-quality mass spectral data is obtained from $\beta$-peptides cleaved from individual beads. Both algorithm-based and manual sequencing confirmed the $\beta$-peptide on the bright bead was indeed $\boldsymbol{\beta 5 3 - 1}$. Notably, while $\beta^{3}$-peptides have been subjected to MS/MS fragmentation before, ${ }^{18}$ our results demonstrate the feasibility of de novo MS/MS decoding of $\beta$-peptide identity from single beads.

To address our initial hypothesis regarding optimization of a nonrecognition face of $\boldsymbol{\beta 5 3 - 1}$, we synthesized a 1000 -member library in which the $\beta^{3}$-homovaline $\left(\beta^{3} \mathrm{~V}\right)$ residues at positions 2 , 5 , and 8 of $\boldsymbol{\beta 5 3 - 1}$ were replaced with one of $10 \beta^{3}$-amino acids (Figure 1). ${ }^{19}$ The library was initially screened under conditions optimized for the control beads, and the 25 brightest beads from a pool of $\sim 8000$ were isolated manually (Figure 2B). ${ }^{9}$ MALDI and MS/MS spectra confirmed that the selected beads possessed among them at least 10 different sequences out of the possible 1000, a hit rate of at least $1 \% .{ }^{19}$ The screen was repeated using $200 \mathrm{nM}{ }^{\text {Bio }} \mathrm{hDM} 2$ (instead of $500 \mathrm{nM}$ ) and five 5-min washes at each washing step (instead of two 2-min washes). ${ }^{9}$ The second screen (Figure 2C) yielded 35 high-signal beads from $\sim 16000$ total beads screened, with only a small proportion of nonhit beads showing even dim Qdots-SA605 fluorescence. MALDI and MS/MS spectra confirmed that these 35 beads possessed among them only three sequences: $\boldsymbol{\beta 5 3 - 8}$ (16 beads), $\boldsymbol{\beta 5 3 - 9}$ (18 beads), and $\boldsymbol{\beta 5 3 - 1 0}$ (1 bead).

Significantly, each of these sequences was identified multiple times among the 25 beads culled from the first screen, confirming that the screen had been successfully tuned to reduce the hit rate from over 1 to $0.3 \%$.

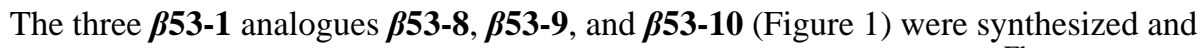
characterized in terms of their ability to inhibit formation of the $\mathrm{p} 53 \mathrm{AD}^{\mathrm{Flu}} \cdot \mathrm{hDM} 2$ complex. ${ }^{1}$ ק53-8 and $\boldsymbol{\beta 5 3 - 9}$, which were well-represented among the hit sequences, both competed with $\mathrm{p} 53 \mathrm{AD}^{\mathrm{Flu}}$ for hDM2 binding, with half-maximal inhibitory concentrations ( $\left(\mathrm{IC}_{50}\right.$ 's) of $13 \pm 1$ and $11 \pm 1 \mu \mathrm{M}$, respectively (Figure 3A). These values represent a roughly 8-fold improvement in inhibitory potency over the parent $\beta$-peptide $\beta$ 53-1. By contrast, $\beta$ 53-10, which was identified only once in the second, more stringent screen, was less soluble in competition assays but appeared to possess an $\mathrm{IC}_{50}$ of $\sim 25 \mu \mathrm{M}$.

Overall secondary structure of the novel analogues was assessed by circular dichroism (CD) spectroscopy. ${ }^{1,21} \beta \mathbf{5 3 - 8}$ and $\beta$ 53-9 (but not $\beta$ 53-10) possessed surprisingly intense 14-helix CD signatures in aqueous solution, with mean residue ellipticities at $214 \mathrm{~nm}$ of -13700 and $-14900 \mathrm{deg} \cdot \mathrm{cm}^{2} \cdot \mathrm{res}^{-1} \cdot ; \mathrm{dmol}^{-1}$, respectively. ${ }^{9}$ These minima are roughly $50 \%$ more intense than that of $\beta$ 53-1. The implied increase in 14-helix structure observed for $\beta \mathbf{5 3 - 8}$ and $\beta \mathbf{5 3 - 9}$ is not unexpected for replacement of $\beta^{3} \mathrm{~V}$ with $\beta^{3}$-homoisoleucine $\left(\beta^{3} \mathrm{I}\right){ }^{21}$ To obtain higher resolution information on the differences between $\beta$ 53-1 and $\beta$ 53-8, we determined the NMR solution structure of $\beta$ 53-8 in methanol in a manner analogous to previous structural work on $\beta$ 53-1. ${ }^{2}$ The NMR structures of $\beta$ 53-8 and $\beta$ 53-1 are strikingly similar, with one major 
difference: inclusion of three $\beta^{3}$ I residues in place of $\beta^{3} \mathrm{~V}$ broadens the corresponding 14-helix face, pushing the $\mathrm{C}_{\beta}$ atoms of residues 2 and 8 from under $9.5 \AA$ apart to over $10.4 \AA$ apart (Figure 3B). This broadening effectively compresses the recognition face: the $\mathrm{C}_{\beta}$ atoms of residues 3 and 9 are at least $10.1 \AA$ apart in $\beta$ 53-1, but under $9.3 \AA$ apart for $\beta$ 53-8 (Figure $3 C$ ). The structure of $\beta$ 53-8 thus provides a clear rationalization for the observed improvement in activity. Furthermore, because the structural differences between $\beta$ 53-8 and $\beta$ 53-1 are so subtle, combinatorial techniques such as those described herein may be especially fruitful for the discovery and refinement of $\beta$-peptides as inhibitors of protein-protein interactions.

In sum, we have reported the first method for synthesizing and screening large $\beta$-peptide libraries. Our techniques for synthesis, screening, and decoding are scalable and immediately compatible with fluorescence-based bead sorters, high-throughput mass spectrometry, and de novo peptide sequencing algorithms. ${ }^{9}$ The output signal can be tuned in multiple ways to achieve a desired hit rate, which should allow for rapid discovery of high-affinity ligands for a wide variety of protein targets.

\section{Supplementary Material}

Refer to Web version on PubMed Central for supplementary material.

\section{Acknowledgments}

We thank Eugene Davidov (Yale Center for Functional Genomics and Proteomics) and Erol Gulcicek (W. M. Keck Biotechnology Resource Laboratory) for MS/MS assistance. This work was supported by the NIH (GM65453 and GM74756), the National Foundation for Cancer Research, and in part by a grant to Yale University, in support of A.S., from the Howard Hughes Medical Institute. N.W.L. was supported by NIH-NRSA GM 074401. J.A.K. is grateful to the NSF for a predoctoral fellowship.

\section{References}

1. Kritzer JA, Lear JD, Hodsdon ME, Schepartz A. J Am Chem Soc 2004;126:9468-9469. [PubMed: 15291512]

2. Kritzer JA, Hodsdon ME, Schepartz A. J Am Chem Soc 2005;127:4118-4119. [PubMed: 15783163]

3. Kussie PH, Gorina S, Marechal V, Elenbaas B, Moreau J, Levine AJ, Pavletich NP. Science 1996;274:948-953. [PubMed: 8875929]

4. One-bead-one-compound libraries have been used extensively for ligand discovery using $\alpha$-peptides, peptidomimetics, and some classes of small molecules. For reviews, see: (a) Lam KS, Lebl M, Krchnak V. Chem Rev 1997;97:411-448. [PubMed: 11848877] (b) Lam KS, Lehman AL, Song AM, Doan N, Enstrom AM, Maxwell J, Liu RW. Methods Enzymol 2003;369:298-322. [PubMed: 14722961]

5. Murray JK, Farooqi B, Sadowsky JD, Scalf M, Freund WA, Smith LM, Chen J, Gellman SH. J Am Chem Soc 2005;127:13271-13280. [PubMed: 16173757]

6. Guichard G, Abele S, Seebach D. Helv Chim Acta 1998;81:187-206.

7. Arvidsson PI, Frackenpohl J, Seebach D. Helv Chim Acta 2003;86:1522-1553.

8. Murray JK, Gellman SH. Org Lett 2005;7:1517-1520. [PubMed: 15816741]

9. See the Supporting Information.

10. Murphy JE, Uno T, Hamer JD, Cohen FE, Dwarki V, Zuckermann RN. Proc Natl Acad Sci USA 1998;95:1517-1522. [PubMed: 9465047]

11. Figliozzi GM, Goldsmith R, Ng SC, Banville SC, Zuckermann RN. Comb Chem 1996;267:437-447.

12. Alluri PG, Reddy MM, Bachhawat-Sikder K, Olivos HJ, Kodadek T. J Am Chem Soc 2003;125:13995-14004. [PubMed: 14611236]

13. Burkoth TS, Beausoleil E, Kaur S, Tang DZ, Cohen FE, Zuckermann RN. Chem Biol 2002;9:647654. [PubMed: 12031671]

14. Humet M, Carbonell T, Masip I, Sanchez-Baeza F, Mora P, Canton E, Gobernado M, Abad C, PerezPaya E, Messeguer A. J Comb Chem 2003;5:597-605. [PubMed: 12959560] 
15. Cho CY, Youngquist RS, Paikoff SJ, Beresini MH, Hebert AR, Berleau LT, Liu CW, Wemmer DE, Keough T, Schultz PG. J Am Chem Soc 1998;120:7706-7718.

16. Olivos HI, Bachhawat-Sikder K, Kodadek T. ChemBioChem 2003;4:1242-1245. [PubMed: 14613120]

17. Control experiments in which ${ }^{\mathrm{Bio}} \mathrm{hDM} 2$ was replaced with $\mathrm{hDM} 2{ }_{1-188},{ }^{\mathrm{Bio}} \mathrm{BSA}$, BSA, or no protein resulted in no observable Qdots-SA605 fluorescence localized to the beads. See the Supporting Information for details.

18. Schreiber JV, Quadroni M, Seebach D. Chimia 1999;53:621-626.

19. Of 20 individual library beads selected at random and all subsequent beads picked as hits, $95 \%$ possessed a single major product in the expected mass range when analyzed by MALDI and MS/ MS.

20. Koradi R, Billeter M, Wuthrich K. J Mol Graphics 1996;14:51-55.

21. Kritzer JA, Tirado-Rives J, Hart SA, Lear JD, Jorgensen WL, Schepartz A. J Am Chem Soc 2005;127:167-178. [PubMed: 15631466] 

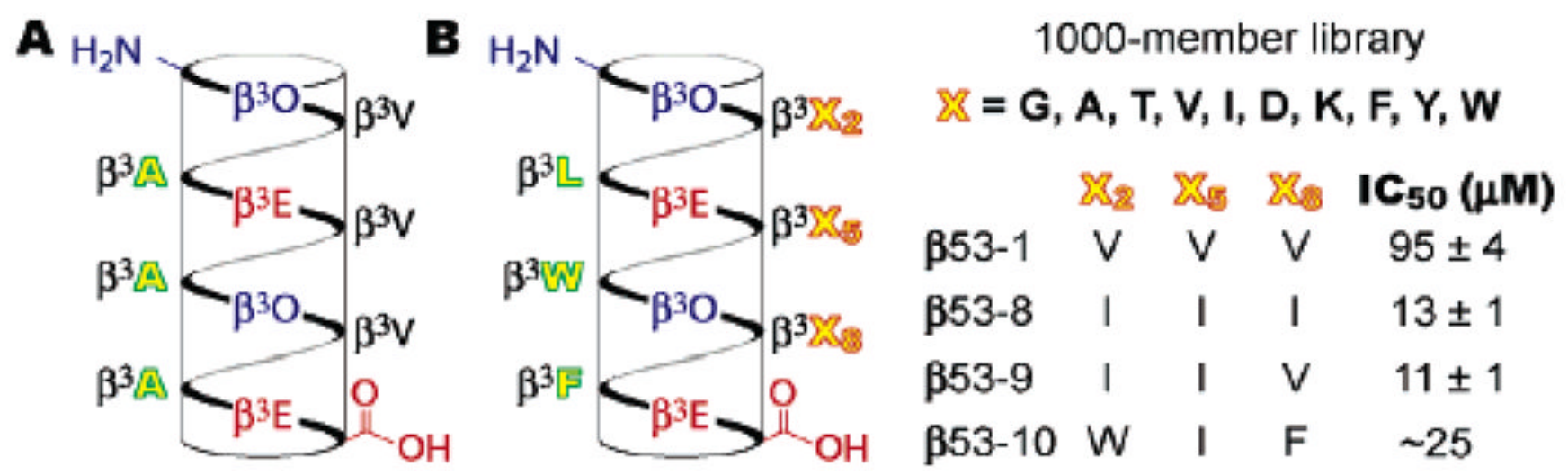

Figure 1.

Helical net representations of (A) $\beta$ NEG and (B) the 1000 -member library, $\beta \mathbf{5 3 - 1}$, and $\beta \mathbf{5 3 - 8}-$ 10. $\beta^{3} \mathrm{X}$ denotes a $\beta^{3}$-homoamino acid; $X$ is the common one-letter code for the analogous $\alpha$ amino acid. Side chains on the recognition, variable, and salt-bridge faces are colored green, orange, and blue/red, respectively. $\mathrm{IC}_{50}$ values are derived from curve fits to the p53AD.hDM2 inhibition data in Figure 3A. 

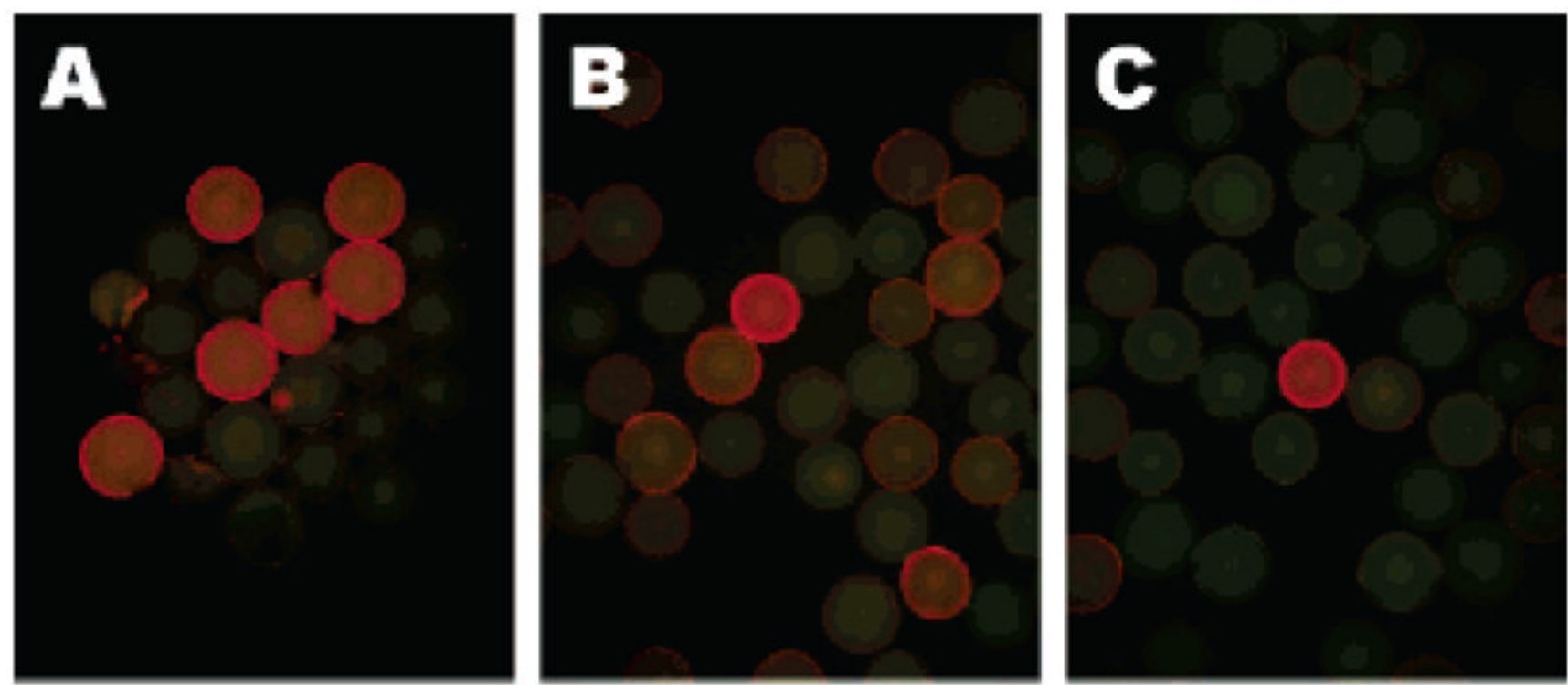

Figure 2.

Representative fluorescence micrographs of $\mathrm{OBO} \beta$ screen. (A) Mixture of $\boldsymbol{\beta 5 3 - 1}$ and $\boldsymbol{\beta}$ NEG beads treated with $500 \mathrm{nM}{ }^{\text {Bio }} \mathrm{hDM} 2$ and $5 \mathrm{nM}$ QDots-SA605 in BW buffer. (B) Beads from the 1000-member library treated as in (A) in BW+ buffer or (C) with $200 \mathrm{nM}{ }^{\text {Bio }} \mathrm{hDM} 2$ and 5 nM QDots-SA605 in BW+ buffer and washed extensively. 

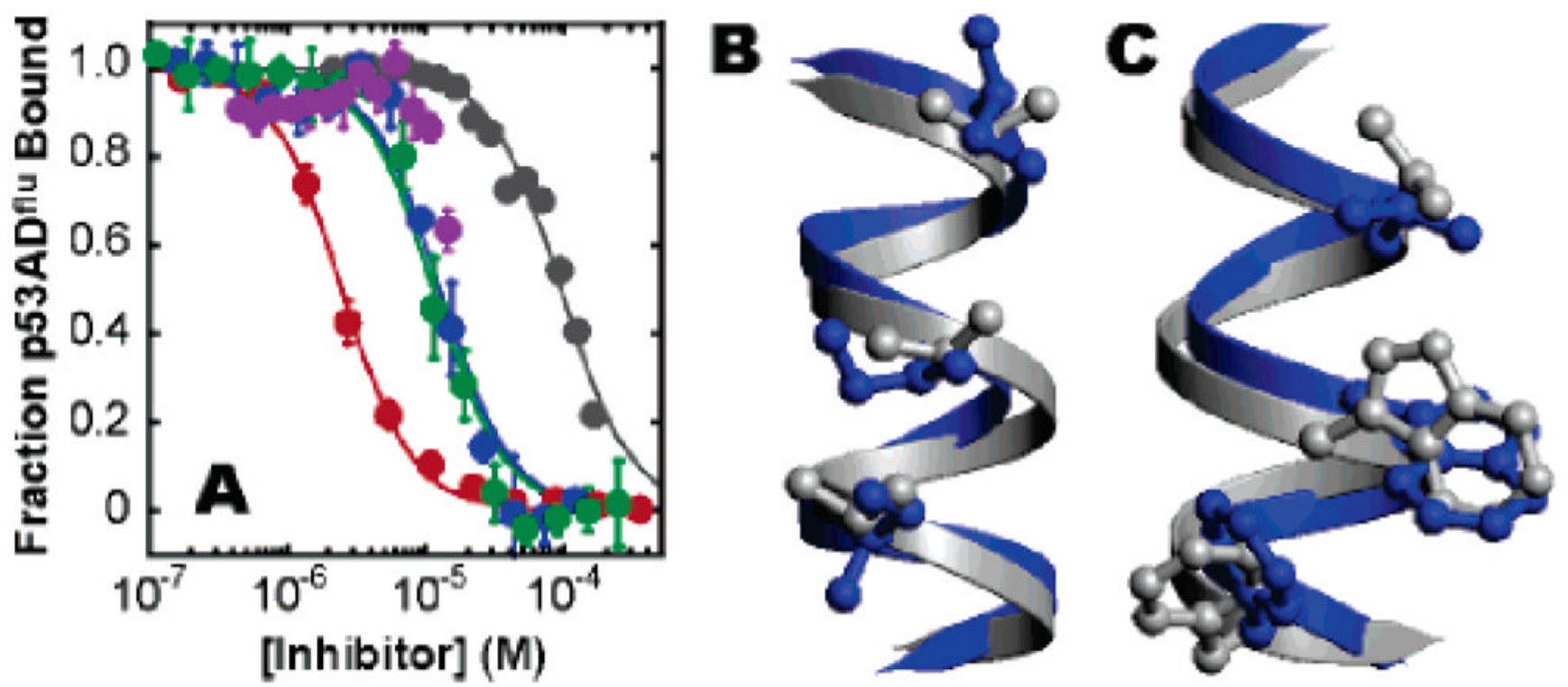

Figure 3.

(A) Fluorescence polarization analysis of the inhibition of p53AD ${ }_{15-31}$ Flu.hDM2 ${ }_{1-188}$ complexation by p53AD ${ }_{15-31}$ (red), $\boldsymbol{\beta 5 3 - 1}$ (gray), $\boldsymbol{\beta 5 3 - 8}$ (blue), $\boldsymbol{\beta 5 3 - 9}$ (green), or $\boldsymbol{\beta 5 3 - 1 0}$ (purple). (B,C) Superposition of the solution structures of $\boldsymbol{\beta 5 3 - 1}$ (gray) and $\boldsymbol{\beta 5 3 - 8}$ (blue) highlighting the varied nonrecognition face $(\mathrm{B})$ and the recognition face $(\mathrm{C}){ }^{20}$ 